\title{
Throughput Optimization in Wireless Multihop Networks with Successive Interference Cancellation
}

\author{
Patrick Mitran , Catherine Rosenberg, Samat Shabdanov \\ Electrical and Computer Engineering Department \\ University of Waterloo
}

\begin{abstract}
Successive Interference Cancellation (SIC) is a potentially powerful technique for improving the performance of wireless multihop networks. This work presents a method to compute the maximum achievable throughput of such networks. We consider the case of a network that uses conflict-free scheduling and has multi-rate and multi-power capabilities. We also consider the case of different levels of SIC, i.e., we will denote by SIC-n a technique in which a receiver can possibly decode up to $n$ signals at a time. We formulate a flexible framework to quantify the throughput improvement that can be obtained in a realistic size multihop network by using SIC- $n$ for $n=2,3$. The optimization framework is formulated as a joint routing, scheduling, and SIC problem under the physical layer interference model for any multihop network and common utility functions.

This joint optimization problem is then numerically solved for max-min throughput for several cases of interest and insights are provided into the gains that can be provided with SIC- $n$ in the case of mesh networks with multi-rate and multi-power capabilities. Specifically, we find that, not surprisingly, when enabling SIC at each node, very significant throughput gains at high transmission power can be obtained. We find that at low transmission power, gains in the range of $25-40 \%$ are possible with SIC-2 at each node and, when in addition the flow pattern is symmetrical, SIC-3 does not bring significant gains over SIC-2. Moreover, compared to mesh networks without SIC, where in the high power regime single-hop transmission to the gateway is optimal, with SIC at each node, this is not necessarily the case. We also show that performing SIC only at the gateway enables non-negligible gains in a multi-hop context. We believe that this study can be useful to network operators to quantify the gains that SIC can provide in a managed mesh network.
\end{abstract}

Index Terms-Multihop Wireless Networks, Scheduling, Successive Interference Cancellation.

\section{INTRODUCTION AND BACKGROUND}

Successive Interference Cancellation (SIC) is a technique that allows a wireless receiver to decode multiple signals successively to either cancel interference or receive more than one packet at a time. We will denote by SIC- $n$ a technique in which a receiver can possibly decode up to $n$ signals at a time. Typically $n=2,3$. More precisely, referring to Figure 1 in a single channel system without SIC, a half-duplex wireless node $i$ can only decode a signal from node $j$ if its SINR (Signal to Interference plus Noise Ratio) is greater than a certain threshold (see later). Hence, if a node $a$ transmits to $b$ at the same time as $j$ transmits to $i$ and if $a$ is closer to $i$ than $j$ is, then $i$ cannot typically decode correctly the signal from $j$. Now if SIC-2 is enabled, then it might be possible for $i$ to first decode the signal from $a$. Node $i$ can then subtract it from its compound received signal so that it can now decode

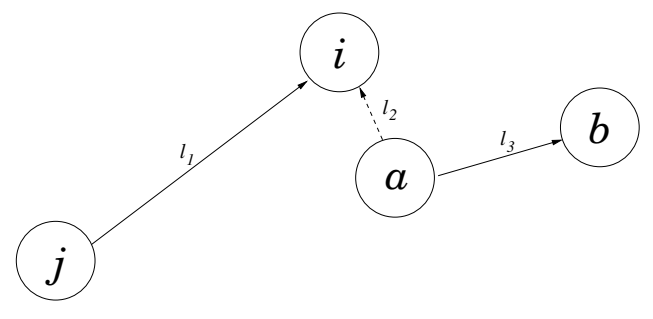

Fig. 1. Illustration of SIC-2

the signal from $j$. In that case, $i$ has cancelled the interference from $a$ to decode the signal from $j$ successfully. Note that if both $j$ and $a$ were trying to send a packet to $i$, then with this technique, $i$ could possibly decode both of them while without this technique, most of the time, $i$ would be unable to decode any of them. The definition of SIC- $n$ will be formalized later.

SIC is a fundamental technique in modern point-tomultipoint systems such as CDMA based cellular networks. This is because in modern cellular systems, interference, more than any other effect, limits performance [1]. In a singlehop TDMA system, SIC would make sense not to reduce interference but to allow the base-station to decode more than one packet at a time. Typically in a single-hop setting, SIC is performed only at the base-station and hence would help for uplink communications.

Successive interference cancellation is known to be a capacity achieving technique for certain types of problems. Specifically, for the information theoretic multiple access channel, it is known that a SIC based approach can achieve the corner points of the capacity region, with time-sharing used to then achieve the entire region [5]. For the 2-user interference channel, in the regime of sufficiently strong interference, it is optimal for each receiver to first decode the interfering transmission, cancel the interference and then decode each receiver's actual intended signal [4]. SIC is also optimal for the single antenna information theoretic broadcast channel [3].

From a more practical side, SIC is perhaps best known for its application to CDMA receivers [11]. SIC has also been proposed for decoding concatenated space-frequency block coding schemes [9] and the complexity of a MIMO-OFDM SIC-based receiver is considered in [8]. SIC has also been proposed for distributed sensor network detection [2].

In this paper, we are interested in studying the performance of TDMA-based (conflict-free) scheduled multi-hop networks 
when SIC is enabled at all nodes or at some nodes. Schedulingbased networks are important since most of the new high data rates wireless technologies, such as WiMax and LTE, have an option for scheduling. Moreover, the achievable throughput for scheduling-based MAC can serve as an upper bound for any random access MAC [7]. Typically in a TDMA-based singlehop cell, all nodes transmit to the base-station (not directly to one another) and hence such a system is not interferencelimited but is rather "base-station-limited" in that the basestation being half-duplex, cannot transmit multiple packets at once (except through sectoring) and cannot receive more than one packet at once. If SIC- $n$ is enabled at the base-station, it could improve the performance of such system by allowing the scheduling of up to $n$ uplink transmissions at once as long as the SINR conditions (to be defined later) are met. Note that enabling SIC at the nodes would not help in that case. In a multihop network, the network performance is limited by both interference and the capability of the nodes (in particular the base-station) and thus, a technique such as successive interference cancellation which directly counters some of the effects of interference and allows multiple decodings should be very well suited to improve performance. However, to the best of our knowledge, there has been no studies quantifying the potential throughput gains that can be obtained by using SIC$n$ in a realistic-sized multihop network. This is the purpose of this paper.

A few words on the interference model are in order. There are many models for wireless channel interference in the networking community. Specifically, three of the most common are the additive interference model, the protocol model and the interference range model [6]. As successive interference cancellation requires proper modeling of the interference generated by all other nodes, only the additive interference model, also referred to as the SINR-based interference model, is compatible with SIC.

To the best of the authors knowledge, only [12] considers applying SIC to TDMA-based multihop networks. In the work there, capacity regions for multi-hop routing with concurrent transmission and SIC are investigated and the concept of rate matrices, which is similar to the notion of ISets defined later in this paper, is used. For multi-hop cellular networks with SIC, only small configurations involving 6 nodes were considered and the throughput on each link was given by Shannon formula $\log (1+$ SINR $)$. In contrast, in the work considered here, SIC was studied for much larger networks with up to 25 nodes and with multi-rate and multi-power capabilities. This was made possible by the formulation of a powerful framework and the development of an efficient computational tool. Furthermore, important engineering insights into the gain quantification due to SIC (as a function of the number of iterations of SIC) in medium sized wireless networks were obtained.

In this paper, we study the optimal joint routing and scheduling configuration under the SINR-based interference model in a multihop network in which SIC- $n$ is enabled. While our formulations are very general in terms of network structure and flow patterns, when deriving numerical results we will mainly focus on managed wireless mesh networks that are configured offline by their network operator to obtain the best throughput performance. A mesh network is composed of a gateway (we will use the terms base-stations and gateway interchangeably) and multiple fixed mesh routers that aggregate the traffic from the end-users. The flow pattern is typically from each router to the gateway (uplink) and from the gateway to each router (downlink). The users and their mobility are not modeled in our framework. We define by network configuration the selection of the routing and scheduling parameters and possibly other parameters (such as power levels and modulation schemes) depending on the problem at hand. The main contributions of the paper can be summarized as follows:

- We formulate a joint routing, scheduling, and SIC-n problem for a general objective function in a multihop wireless network with multi-rate and multi-power capabilities. This formulation does not make any assumptions on the flow pattern or the network structure. Instances of this problem exist for the case where SIC is available only at the gateway or at all nodes.

- We then define the performance of the network as a max-min throughput since as discussed in [7] and [10], it is a reasonable objective in the case of a managed mesh network. Then the problem turns out to be a very large NP-hard Linear Program (LP).

- We develop an efficient tool to compute numerically exact solutions for small to medium-size networks in reasonable time.

- Our formulation enables us to quantify the gains that can be obtained by using SIC- $n$ for realistic-size networks. In particular, we show that SIC- $n$ can achieve significant throughput gains even at low powers as compared to the case where SIC is not enabled and that at low to medium power, SIC-3 does not bring significant gains above SIC2.

- We show that even in a multihop network, restricting SIC$n$ to be used only at the gateway can bring non-negligible gains.

- We also show that, at high transmission power, singlehopping is not optimal when SIC is enabled. Indeed significant throughput improvement can be obtained by allowing multihopping even if single-hopping from all nodes to the gateway is feasible.

The rest of this paper is structured as follows. In Section II, we provide the network model and the corresponding optimization problem. In Section III, we provide some numerical results and further insights into joint scheduling and routing with SIC, while Section IV concludes this paper.

\section{Network Model and Problem Formulation}

\section{A. Network Model}

A multihop network consists of a set of nodes $\mathcal{N}$. Each node is equipped with a set of coding and modulation schemes which results in a set of possible transmission rates $\mathcal{R}=$ 
$\left\{r_{1}, \ldots r_{|\mathcal{R}|}\right\}$. Each transmission rate $r \in \mathcal{R}$ can only be decoded at the receiver with an acceptable bit-error (or frameerror) rate provided the received SINR is above a threshold $\beta(r)$. Each node may also use any one of $\mathcal{P}=\left\{P_{1}, \ldots, P_{|\mathcal{P}|}\right\}$ transmission powers and may use a different modulation and a different transmit power at different times.

A link $\ell$ is then defined by a 4-tuple $l=$ $(o(\ell), d(\ell), P(\ell), r(\ell))$, where $o(\ell)$ is the source node of the link, $d(\ell)$ is the destination of the link, $P(\ell) \in \mathcal{P}$ is the power employed on link $\ell$ and $r(\ell) \in \mathcal{R}$ is the rate of link $\ell$ and $o(\ell) \neq d(\ell)$. In the absence of interference, a link $\ell$ is called feasible if its SNR (then given by $P(\ell) G_{o(\ell), d(\ell)} / N_{0}$ ) is greater or equal to the threshold $\beta(r(\ell))$, where $G_{o(\ell), d(\ell)}$ is the channel power gain between node $o(\ell)$ and $d(\ell)$ and $N_{0}$ is the noise power at receiver $d(\ell)$. Let $\mathcal{L}$ be the set of all possible feasible links.

In general, not all links in the network can be simultaneously active. A set of links that can be simultaneously active without creating harmful interference to each other, is said to form an ISet (short for Independent Set). More precisely, a set of links $s \subset \mathcal{L}$ is an ISet only if it obeys the three following necessary conditions:

[C1] If $\ell_{1}, \ell_{2} \in s$ and $\ell_{1} \neq \ell_{2}$, then $o\left(\ell_{1}\right) \neq o\left(\ell_{2}\right)$,

[C2] if $\ell_{1}, \ell_{2} \in s$ and $\ell_{1} \neq \ell_{2}$, then $o\left(\ell_{1}\right) \neq d\left(\ell_{2}\right)$,

[C3] if $\ell_{1}, \ell_{2} \in s$ and $\ell_{1} \neq \ell_{2}$, then $d\left(\ell_{1}\right) \neq d\left(\ell_{2}\right)$.

The first condition implies that two simultaneously active links cannot have the same origin, while the second condition is the half-duplex constraint which does not allow for a node to simultaneously transmit and receive. The third condition implies that a node cannot receive multiple transmissions at once.

In the absence of successive interference cancellation abilities at any nodes, a set $s \subset \mathcal{L}$ is an ISet if it satisfies [C1], [C2] and [C3] above and in addition,

[S1] For each $\ell \in s$,

$$
\frac{P(\ell) G_{o(\ell), d(\ell)}}{N_{0}+\sum_{\ell_{1} \in s, \ell_{1} \neq \ell} P\left(\ell_{1}\right) G_{o\left(\ell_{1}\right), d(\ell)}} \geq \beta(r(\ell)) .
$$

This is simply the requirement that each receiver in the ISet has an SINR of at least $\beta(r(l))$.

The set of all ISets determined by conditions [C1], [C2], [C3] and [S1] is denoted by $\mathcal{I}_{1}$.

If successive interference cancellation of level 2 (i.e., SIC2 ) is allowed, then a node may either decode the intended transmission directly provided the SINR is high enough, or it may first attempt to decode an interfering transmission first, cancel the interference thus caused, and then attempt to decode the intended transmission. Note that if the first interfering transmission is intended for that node, then it can receive 2 packets at once. With this approach, a set of links $s \subset \mathcal{L}$ is feasible if it satisfies [C1], [C2], and in addition

[C4] for each $\ell \in s, \sum_{\ell_{1} \in s} 1_{\left\{d\left(\ell_{1}\right)=d(\ell)\right\}} \leq 2$,

[S2] For each $\ell \in s$, either condition (1) is valid or, there is an $\ell_{1} \neq \ell$ such that

$$
\begin{gathered}
\frac{P\left(\ell_{1}\right) G_{o\left(\ell_{1}\right), d(\ell)}}{N_{0}+\sum_{\ell_{2} \in s, \ell_{2} \neq \ell_{1}} P\left(\ell_{2}\right) G_{o\left(\ell_{2}\right), d(\ell)}} \geq \beta\left(r\left(\ell_{1}\right)\right) \\
\frac{P(\ell) G_{o(\ell), d(\ell)}}{N_{0}+\sum_{\ell_{2} \in s, \ell_{2} \neq \ell, \ell_{1}} P\left(\ell_{2}\right) G_{o\left(\ell_{2}\right), d(\ell)}} \geq \beta(r(\ell)) .
\end{gathered}
$$

The set of ISets determined by conditions [C1], [C2], [C4] and [S2] is denoted by $\mathcal{I}_{2}$. Note that $\mathcal{I}_{1} \subset \mathcal{I}_{2}$.

In this paper, we will also consider the case of 3 successive decodings. Analogously to conditions [C4] and [S2], we have the following:

[C5] for each $\ell \in s, \sum_{\ell_{1} \in s} 1_{\left\{d\left(\ell_{1}\right)=d(\ell)\right\}} \leq 3$,

[S3] For each $\ell \in s$, either condition (1) is valid or, there is an $\ell_{1} \neq \ell$ such that conditions (2) and (3) are valid or there are distinct $\ell_{1}, \ell_{2} \neq \ell$ such that

$$
\begin{aligned}
& \frac{P\left(\ell_{2}\right) G_{o\left(\ell_{2}\right), d(\ell)}}{N_{0}+\sum_{\ell_{3} \in s, \ell_{3} \neq \ell_{2}} P\left(l_{3}\right) G_{o\left(\ell_{3}\right), d(\ell)}} \geq \beta\left(r\left(\ell_{2}\right)\right) \\
& \frac{P\left(\ell_{1}\right) G_{o\left(\ell_{1}\right), d(\ell)}}{N_{0}+\sum_{\ell_{3} \in s, \ell_{3} \neq \ell_{1}, \ell_{2}} P\left(\ell_{2}\right) G_{o\left(\ell_{2}\right), d(v)}} \geq \beta\left(r\left(\ell_{1}\right)\right) \\
& \frac{P(\ell) G_{o(\ell), d(\ell)}}{N_{0}+\sum_{\ell_{3} \in s, \ell_{3} \neq \ell, \ell_{1}, \ell_{2}} P\left(\ell_{2}\right) G_{o\left(\ell_{2}\right), d(\ell)}} \geq \beta(r(\ell)) .
\end{aligned}
$$

The set of ISets determined by conditions [C1], [C2], [C5] and [S3] is denoted by $\mathcal{I}_{3}$. Note that $\mathcal{I}_{1} \subset \mathcal{I}_{2} \subset \mathcal{I}_{3}$.

\section{B. Joint Routing and Scheduling Optimization Problem}

Given one set of ISets $\mathcal{I}$ (say $\mathcal{I}=\mathcal{I}_{2}$ ), a link schedule is an $|\mathcal{I}|$-dimensional vector $\alpha=\left\{\alpha_{s}\right\}_{s \in \mathcal{I}}$, which assigns a weight $\alpha_{s} \geq 0$ to each ISet $s \in \mathcal{I}$. Each $\alpha_{s}$ is interpreted as the fraction of time that each ISet $s$ is scheduled. Then necessarily $\sum_{s \in \mathcal{I}} \alpha_{s}=1$. Let $\mathcal{I}_{\ell}$ be the set of all ISets in $\mathcal{I}$ that contain link $\ell$.

A flow $f$ is defined by an ordered pair of nodes, and for notational convenience, given a flow $f, f_{s}$ and $f_{d}$ denote the start and end node respectively. With each flow $f$, one associates a rate $\lambda_{f}$. Define $\mathcal{F}$ as the set of all flows.

Given $\mathcal{F}, \mathcal{L}, \mathcal{N}$, and $\mathcal{I}$ defined earlier, denote by $x_{f}(\ell)$ the amount of flow $f$ transmitted over a link $\ell$. Let $\boldsymbol{x}_{f}=$ $\left\{x_{f}(\ell)\right\}_{\ell \in \mathcal{L}}$. In order to simplify notation, we introduce the vector $\boldsymbol{x}=\left\{\boldsymbol{x}_{f}\right\}_{f} \in \mathcal{F}$. The vector $\boldsymbol{x}$ represents the aggregated traffic vector of all flows allocated over all links.

Our problem consists of finding a link schedule and an aggregated traffic vector that maximize a given network utility function $U\left(\left\{\lambda_{f}\right\}_{f \in \mathcal{F}}\right)$ (see [7] and [10] for more details in joint routing and scheduling problem in the case without SIC), and this can be formulated as:

$$
\begin{gathered}
\max _{\boldsymbol{\alpha}, \boldsymbol{x}, \lambda} U\left(\left\{\lambda_{f}\right\}_{f \in \mathcal{F}}\right) \\
\sum_{\substack{\ell \in \mathcal{L} \\
o(\ell)=n}} x_{f}(\ell)-\sum_{\substack{\ell \in \mathcal{L} \\
d(\ell)=n}} x_{f}(\ell)=\left\{\begin{array}{ccc}
\lambda_{f}, & n=f_{s} \\
-\lambda_{f}, & n=f_{d} & \forall n \in \mathcal{N} \\
0, & \text { else } & f \in \mathcal{F}
\end{array}\right. \\
\sum_{f \in \mathcal{F}} x_{f}(\ell) \leq r(\ell) \sum_{s \in \mathcal{I}_{\ell}} \alpha_{s}
\end{gathered}
$$




$$
\begin{aligned}
\sum_{s \in \mathcal{I}} \alpha_{s} & =1 \\
\boldsymbol{\alpha}, \boldsymbol{x} & \geq 0
\end{aligned}
$$

Condition (8) specifies the flow conservation constraints. Link scheduling constraints are given in (9) and (10). Specifically, (9) defines the link capacity constraint that the aggregated amount of all flows over the link cannot exceed its scheduled capacity.

Note that this problem does not change when we enable SIC-2 or SIC-3 or disable SIC everywhere, if we enable SIC only at some nodes, etc. The only differences between all these cases is the set of all ISets $\mathcal{I}$. The problem (7)-(11) can be very large since the cardinality of $\mathcal{I}$ scales with the number of links $|\mathcal{L}|$, which is of the order of $O\left(N^{2}\right)$ in a network with $N$ nodes. When the utility function $U$ is linear (e.g., $\left.U\left(\left\{\lambda_{f}\right\}_{f \in \mathcal{F}}\right)=\sum \lambda_{f}\right)$ or $U\left(\left\{\lambda_{f}\right\}_{f \in \mathcal{F}}\right)=\min \left\{\lambda_{f}\right\}$, the problem is a standard LP formulation, yet, it is NP-hard to solve. However [10] proposes efficient algorithmic techniques to compute the optimal solutions for relatively large networks (up to 60 nodes) when the utility function is linear. The optimal solution for this problem provides not only the optimal utility but also the corresponding optimal configuration (scheduling and traffic vectors, power levels and rates). In general, the optimal configuration is not unique. Note that we have not made any assumptions on the flow pattern and hence this formulation is not limited to the flow patterns usually seen in mesh networks.

\section{NumericAl RESUlts}

In this section, we provide exact solutions using our computation tool for mid-size mesh networks. We use $U\left(\left\{\lambda_{f}\right\}_{f \in \mathcal{F}}\right)=\min \left\{\lambda_{f}\right\}$, i.e., the throughput performance is the max - min throughput since as discussed in [7] and [10], it is a reasonable network utility in the case of a managed mesh network. The results below illustrate some of the salient aspects of applying SIC in a mesh network. We define a mesh network as a network with $N$ nodes and one gateway. The flow pattern for mesh networks is typically from each node to the gateway (uplink) and from the gateway to each node (downlink). In practice, a downlink flow has usually a larger rate than an uplink flow. To take this in consideration, we consider a weighted optimization problem. Specifically, in the optimization problem above, we replace condition (7) by:

$$
\begin{gathered}
\max _{\boldsymbol{\alpha}, \boldsymbol{x}, \lambda} \lambda \\
0 \leq \lambda \leq \frac{\lambda_{f}}{w_{f}}, \quad \forall f \in \mathcal{F}
\end{gathered}
$$

where $w_{f}$ is a per flow weighting factor. In this paper, we will consider two types of weighting factors. The first, we call equal weighting and denote as 1:1. It is obtained by choosing all $w_{f}=1$. For the second type of weighting factors, we choose $w_{f}=1$ for all uplink flows and $w_{f}=2$ for all downlink flows, thus ensuring that downlink flows obtain twice the throughput of uplink flows after optimization. We denote this second weighting type by $1: 2$. By solving this optimization problem, we will obtain an optimal network configuration for routing and scheduling such that $\lambda$ is maximized. We will show our results in terms of per node throughput denoted as $\lambda^{T}$, i.e., in the 1:1 case, $\lambda^{T}=2 \lambda$ while in the $1: 2$ case, $\lambda^{T}=3 \lambda$. In the following, we will present results for the case where the flow pattern is uplink-only, downlink-only, or mixed with equal weighting or 1:2 weighting.

For simplicity only, we model the channel power gain by the path-loss factor

$$
G_{o(\ell), d(\ell)}=\left(\frac{d_{o(\ell), d(\ell)}}{d_{0}}\right)^{-\eta},
$$

where $d_{o(\ell), d(\ell)}$ is the distance between nodes $o(\ell)$ and $d(\ell)$, $d_{0}$ is the near-field crossover distance and $\eta$ is the pathloss exponent. Note that our framework is general enough to accommodate any (quasi-static) channel model.

We will focus on the 24 nodes (plus the gateway) network shown in Figure 2 called Net-A in the following. Note that we have run extensive computations for different (random) node positions and different numbers of nodes and we have always seen the same type of qualitative trends except for one trend with respect to low power that is reported later. We assume that $N_{0}=-100 \mathrm{dBm}$, the reference distance $d_{0}=0.1$ and the path loss exponent is $\eta=3$. Except when mentioned otherwise, we assume that all nodes use the same transmit power $P$ and the same single modulation scheme yielding a rate of $r_{1}=1$ with a corresponding SINR threshold $\beta\left(r_{1}\right)=6.4 \mathrm{~dB}$. In that case, the minimum power for network connectivity is $P_{\text {min }}=-31.37 \mathrm{dBm}$, while the minimum power required for each node to be able to communicate directly with the gateway in a single-hop is $P_{S H}=-15.32 \mathrm{dBm}$. We will show all our results by plotting the per node throughput $\lambda^{T}$ as a function of $P \geq P_{\min }$. Note that for each value of $P$, a possibly different set of feasible links exist and hence while the "nodetopology" (i.e., the node positions) remains unchanged, the "link-topology" (i.e., the set of feasible links) varies with $P$.

In Figure 3, we show the max-min rate offered to a node in Net-A as a function of the transmission power $P$ when the flow pattern is uplink-only for five cases. The first is traditional multihop mesh operation without SIC (labeled No SIC), i.e., the set of ISets is $\mathcal{I}_{1}$. The next two other cases correspond to SIC-2 and SIC-3 respectively. The last two cases, labeled SIC-2+SH and SIC-3+SH will be defined and discussed later.

For the network without SIC, the maximum achievable maxmin rate is $1 / 24=0.0417$, which is achieved at a moderate power of $P=-21 \mathrm{~dB}$ thanks to the multihop advantage discussed in [10]. For SIC-2, the theoretical maximum is $2 / 24=0.0833$ as the gateway can now receive up to two simultaneous transmissions. This throughput is achieved, though at considerably higher power than required to achieve the plateau without SIC. For SIC-3, the theoretical plateau is $3 / 24=0.125$, yet at no power setting is this performance achieved as the node-topology does not permit the systematic scheduling of three simultaneous transmissions to the gateway 


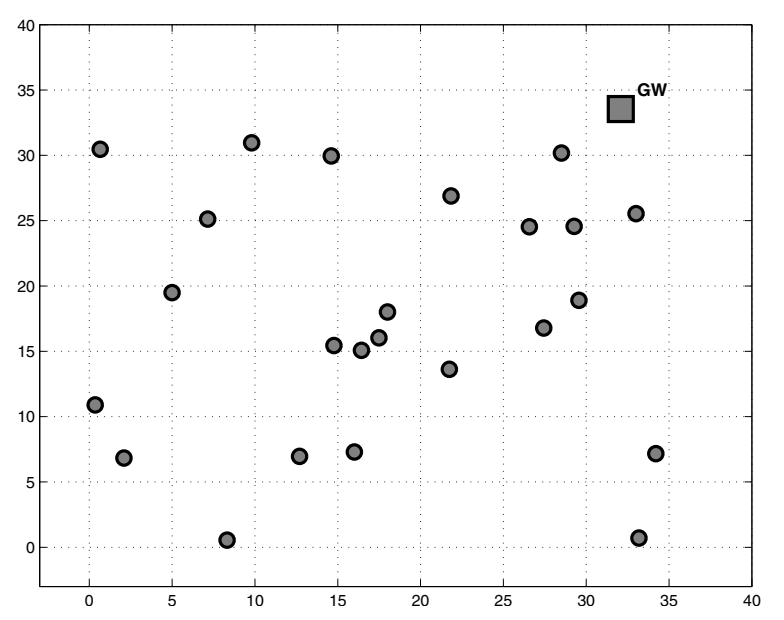

Fig. 2. Placement of nodes in Net-A.

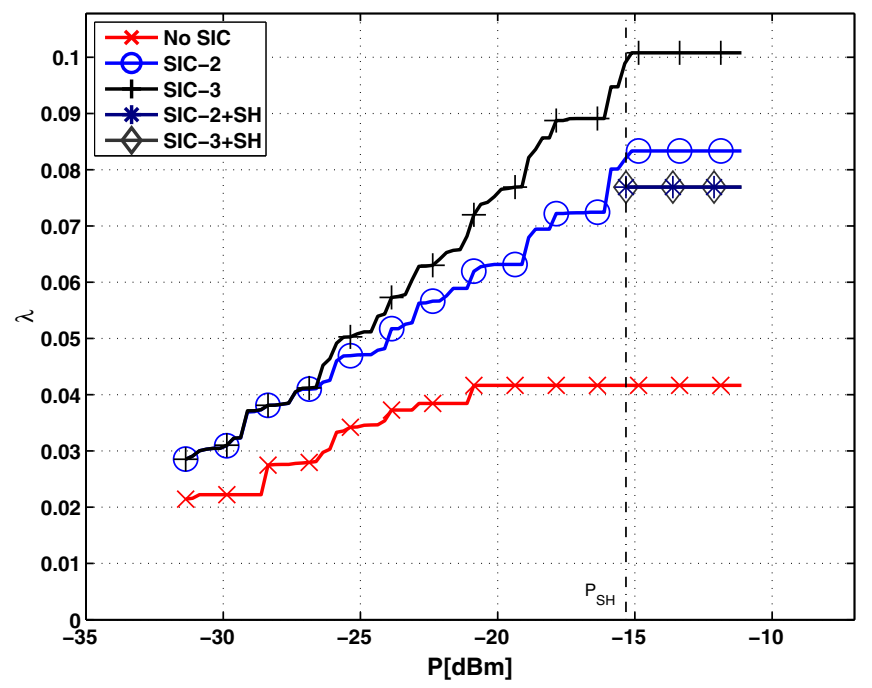

Fig. 3. Max-min rate vs. $P$ in Net-A, uplink-only flow pattern

(and this fact has been observed for other random nodetopologies as well). For this specific node-topology, only half of the additional rate increase that three decodings with SIC could theoretically provide over two decodings is achieved. We also observe that interestingly, at $P=P_{\text {min }}$, i.e., the minimum power for network connectivity, both SIC-2 and SIC-3 provide approximately a $30 \%$ improvement in maxmin throughput compared to no SIC. Furthermore, in the low power regime, while two and three decodings do improve upon no SIC, they provide the same improvement, and thus SIC with two decodings is optimal in this regime (for this nodetopology).

In Figure 4, we show the max-min rate offered to a node in Net-A as a function of the transmission power $P$ when the flow pattern is downlink-only for the same cases. In this case, the optimal max-min rate is limited by the gateway which, if in continuous operation, can only produce a max-min rate

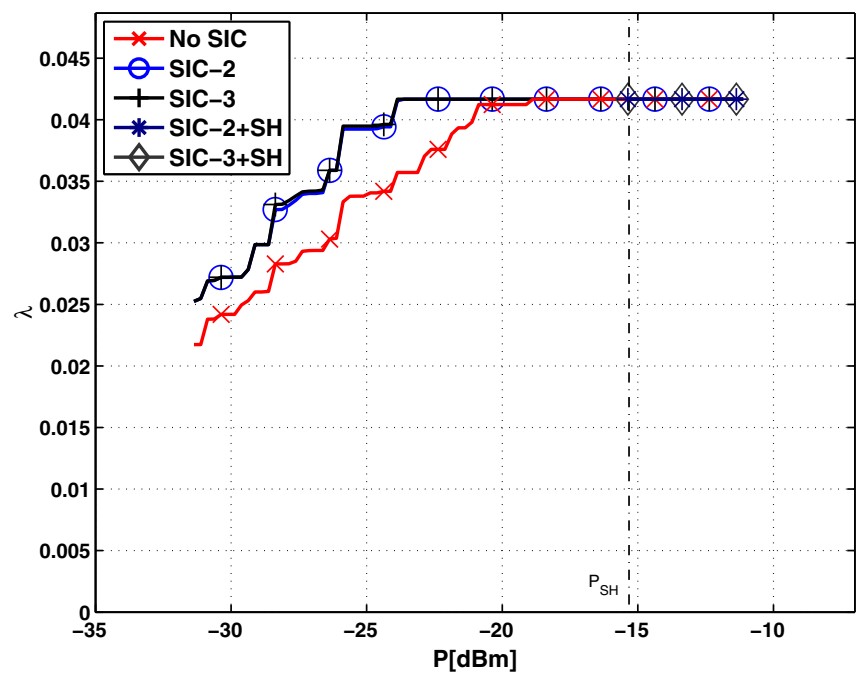

Fig. 4. Max-min rate vs. $P$ in Net-A, downlink-only flow pattern.

of $1 / 24=0.0417$ per node, regardless of the number of decodings applied with SIC. From the figure, we find that at low to medium power, SIC provides higher throughput and the plateau is reached at smaller power settings with SIC than without. Furthermore, three decodings with SIC does not improve upon two decodings with SIC, and thus the lower complexity of two decodings is optimal for this node-topology. As expected, the gains obtained by using SIC on the downlink are less significant than those obtained on the uplink, however they are far from being negligible (about 10-18\%).

In Figure 5, we consider jointly optimizing both uplink and downlink flows for Net-A. The top figure shows the case of equal weighting while the bottom figure show the case where the weighting is $1: 2$. Even, when the uplink weight decreases, i.e., the flow pattern is asymmetrical in rate in favor of the downlink flows (as it is typically in access networks), the gains of using SIC are significant at all power regimes (from 25 to $40 \%$ for the equal weighting case with SIC-2 and 20 to $30 \%$ when the weighting is 1:2). SIC-3 improves the per node max-min throughput only at high transmit power. As mentioned earlier, we have tried many different nodetopologies and found that the qualitative behaviour was always the same except at very low power (i.e., $P \cong P_{\text {min }}$ ). Indeed for some node-topologies, we found no gain when using SIC at very low power.

In Figure 6, we consider performing SIC only at the gateway for Net-A. Specifically, we look at the max-min rate when the flow pattern is the mixed flow pattern with equal weighting when only the GW can perform SIC. Both for SIC-2 and SIC3 , we find a moderate gain in performance at low transmit power when using SIC only at the gateway as opposed to not enabling SIC, while at high power, not surprisingly SIC at the gateway performs very well both for $n=2,3$. We attribute the gap at very high power for SIC-3 between the case where SIC is only enabled at the gateway and the case where SIC 

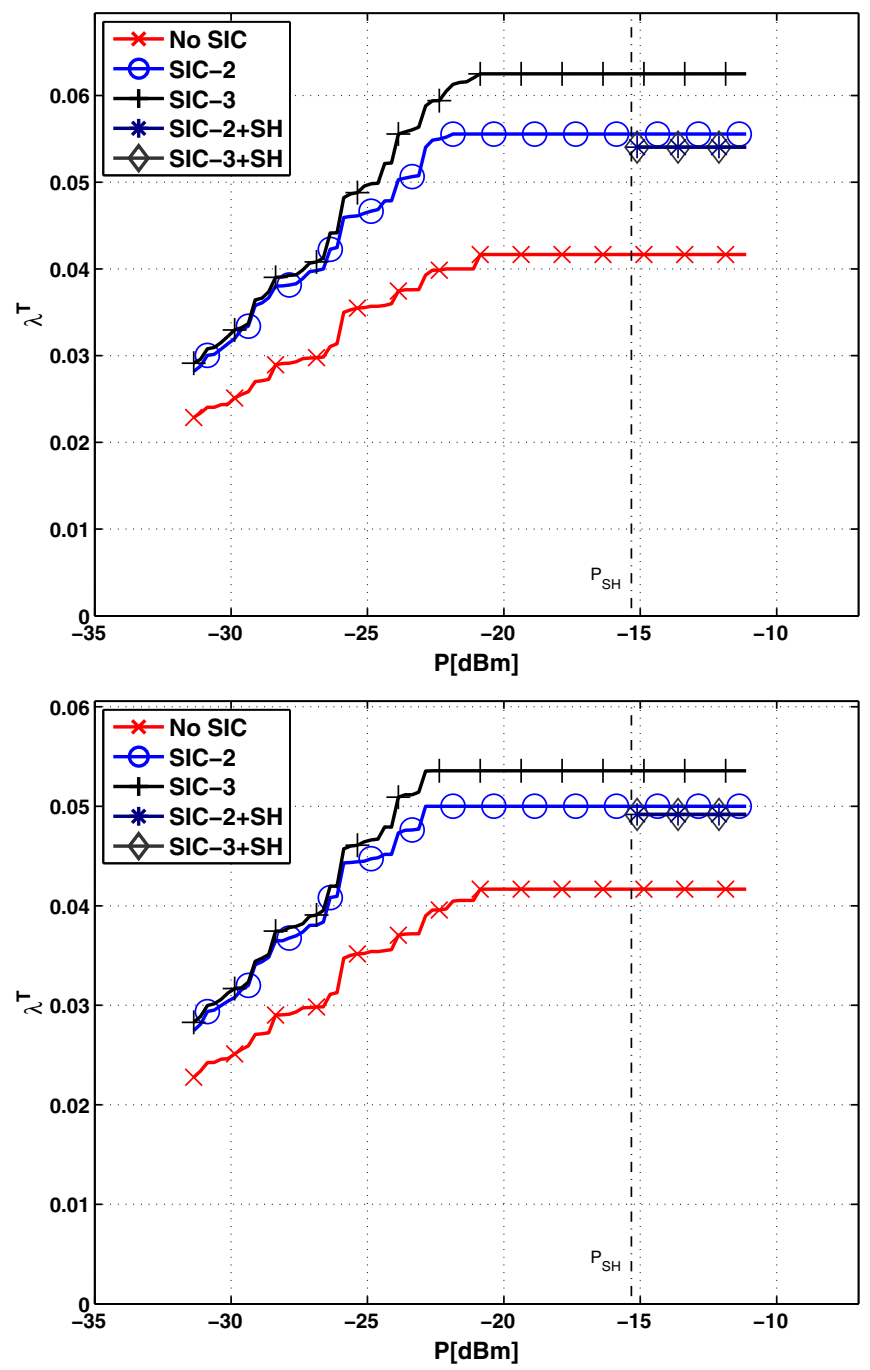

Fig. 5. Max-min rate vs. $P$ in Net-A, mixed flow pattern. Top: equal weighting. Bottom: 1:2 weighting.

is enabled everywhere to the fact that at high power, singlehoping to the gateway is not optimal when SIC is enabled everywhere.

To investigate this further, we focus on the single-hop power regime (i.e., the transmit power $P \geq P_{S H}$ ) and compare the cases when SIC is enabled and single-hop is enforced to the case where multihop is allowed. In our model, we can enforce single-hop by only considering that the direct links from each node to the gateway and from the gateway to each node are feasible. As can be seen in Figures 3, 4 and 5, where the curves labeled SIC- $n+\mathrm{SH}$ correspond to the case where single-hop is enforced, single-hop is not optimal when SIC is enabled everywhere except for the downlink case (as expected) and in fact can be quite sub-optimal in the case of SIC-3.

In Figure 7, we consider the case where 2 modulation schemes are enabled in Net-A, one yielding a rate $c_{1}=1$ with an SINR threshold $\beta_{1}=6.4 \mathrm{~dB}$ and the second one yielding a rate $c_{2}=2$ with an SINR threshold $\beta_{1}=9.4 \mathrm{~dB}$. We compare
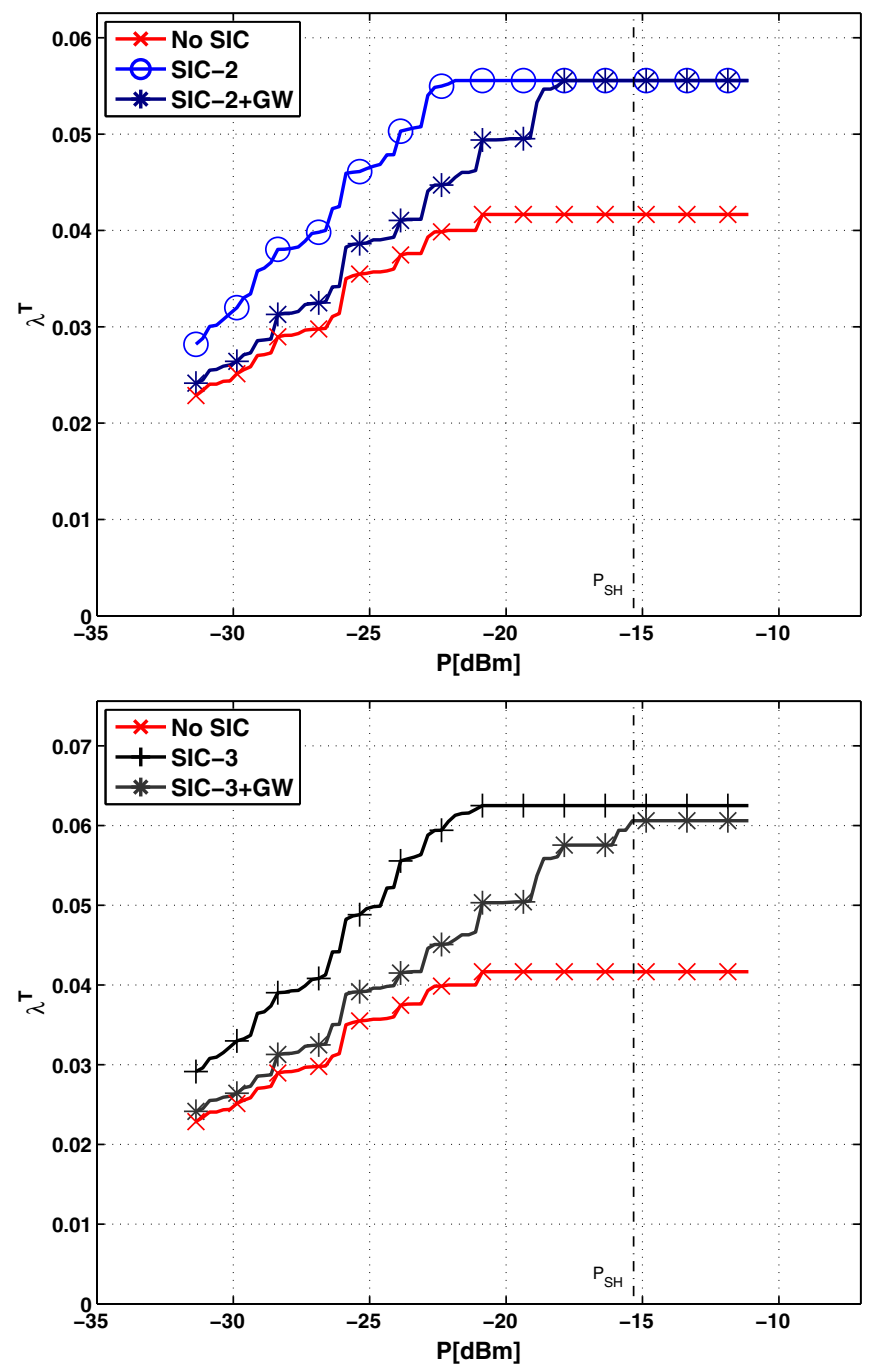

Fig. 6. Max-min rate vs. $P$ in Net-A, equal weighting flow pattern with SIC everywhere or only at the gateway. Top: with SIC-2. Bottom: with SIC-3.

the following three cases, one where only the first modulation is enabled, one where only the second modulation is enabled and one where both modulations are enabled and for each case, we compute the max-min throughput for the case with no SIC and the case with SIC-2. This figure shows how SIC cannot be used as a substitute for multi-rate (since the case with the 2 rates is much better than the case with the lowest rate and SIC), that multi-rate without SIC yields better results (in terms of connectivity and throughput) than the case with the highest rate and that SIC-2 on top of multi-rate brings significant throughput gains when compared to the case with multi-rate and no SIC.

In Figure 8, we consider the benefits of using multiple transmission powers in Net-A. Specifically, we consider two transmission powers, one at $P$ and the second set to $5 \mathrm{~dB}$ lower than $P$. We consider the uplink-only flow pattern and show the max-min throughput as a function of $P$ for the cases with no SIC, SIC-2 and SIC-3 when either a single 


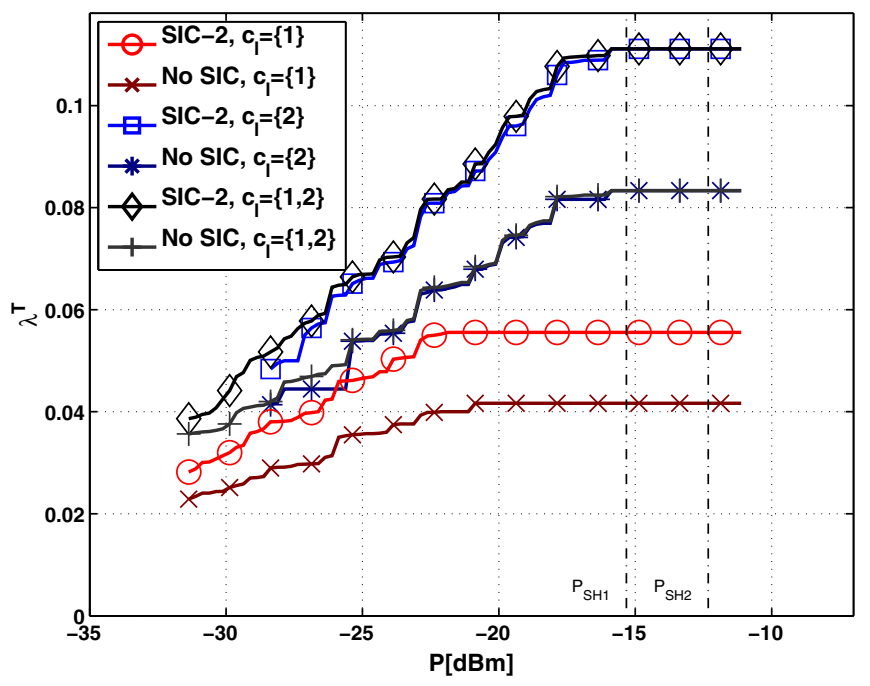

Fig. 7. Max-min rate vs. $P$ in Net-A, equal weighting flow pattern when multi-rate is enabled.

transmit power $P$ is allowed or two transmit powers are allowed ( $P$ and $P-5 \mathrm{~dB}$ ). We find that without SIC or with SIC-2, relatively minor gains are to be had by using these two power settings. Furthermore, the plateau of the throughput in the high power regime is unchanged as it was already at its maximal theoretical value. However, for SIC-3, we find that interestingly, while no significant gain is found over most of the power range, when $P$ is sufficiently high, significant improvements are obtained. We attribute this to the fact that to schedule 3 users simultaneously at the gateway requires a large disparate range of received power at the gateway. While geographic diversity is not enough to provide the full benefit of SIC with 3 decodings, by adding the additional flexibility of two power levels $5 \mathrm{~dB}$ apart, it now becomes possible to schedule many more triples of users, and thus almost achieve the limit of SIC-3.

We are careful not to draw conclusions on the case with multiple power since not enough different values of the offset have been tried to ensure that "power diversity" will not help. In fact, we believe that multiple power levels should greatly help if we have enough granularity. We plan to study this in more details in the future.

\section{CONCLusions}

In this paper, we have quantified the gains obtained by using successive interference cancellation in a multi-hop wireless network. We have found that SIC is a very efficient technique to improve the per node max-min throughput at almost all power ranges. We also found that at high power, single-hop transmission to the gateway is not optimal in the presence of SIC. Furthermore, while significant gains can be provided with SIC, the additional gain provide with 3 decodings over two decodings shows diminishing returns. In addition, performing SIC at the gateway provided some non-negligible gains. Finally using SIC on top of multi-rate brings significant gains.

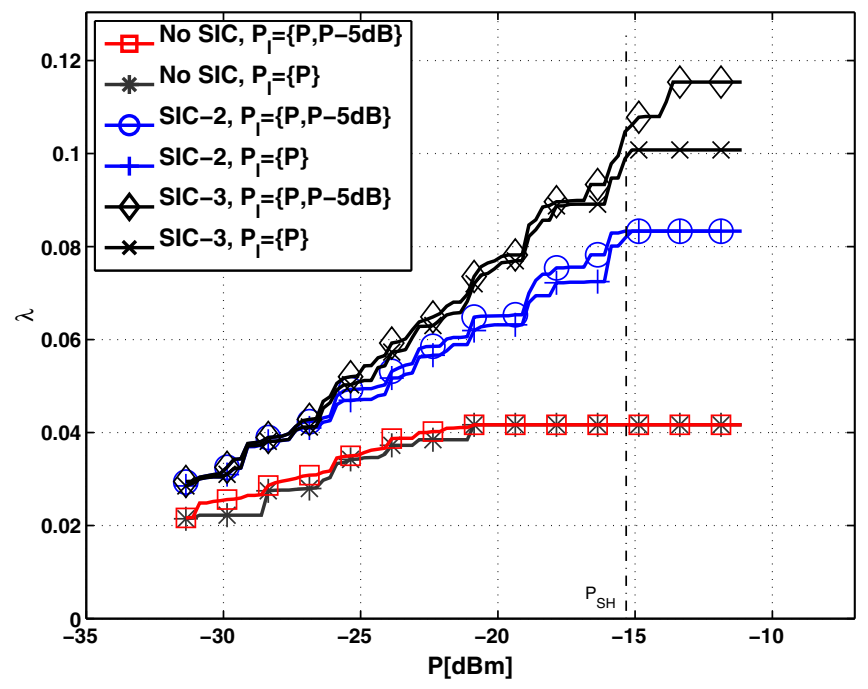

Fig. 8. Max-min uplink rate with 2 transmission powers in Net-A,

\section{REFERENCES}

[1] J. G. Andrews, "Interference cancellation for cellular systems: A contemporary overview," IEEE Wireless Commun. Mag., vol. 12, pp. 19-29, Apr. 2005.

[2] D. Bielefeld and R. Mathar, "Distributed detection with transmit-only sensors and a successive interference cancellation receiver," in IEEE ISSPIT, UAE, 2009, pp. $207-212$.

[3] A. B. Carleial, "A simple converse for broadcast channels with additive white gaussian noise," IEEE Trans. Inform. Theory, vol. 20, no. 2, pp. 279-280, Mar. 1974.

[4] — , "Interference channels," IEEE Trans. Inform. Theory, vol. 24, no. 1, pp. 60-70, Jan. 1978.

[5] T. Cover and J. Thomas, Elements of Information Theory, 2nd ed. New York:Wiley, 2006.

[6] A. Iyer, C. Rosenberg, and A. Karnik, "What is the right model for wireless channel interference?" vol. 8, no. 5, pp. 2662-2671, May 2009.

[7] A. Karnik, A. Iyer, and C. Rosenberg, "Throughput-optimal configuration of fixed wireless networks," IEEE/ACM Transaction in Networking, vol. 16, no. 5, pp. 1161-1174, 2008.

[8] J. Ketonen, M. Juntti, and J. R. Cavallaro, "Performance-complexity comparison of receivers for a LTE MIMO-OFDM system," To appear in IEEE Trans. Signal Processing, 2010.

[9] T. Lai, S. Muruganathan, and A. Sesay, "Performance analysis and multistage iterative receiver design for concatenated spece-frequency block coding schemes," vol. 7, no. 11, pp. 4208 - 4214, Nov. 2008.

[10] J. Luo, C. Rosenberg, and A. Girard, "Engineering wireless mesh networks: Joint scheduling, routing, power control and rate adaptation," To appear in IEEE/ACM Trans. Networking, 2010.

[11] P. Patel and J. Holtzman, "Analysis of a simple successive interference cancellation scheme in a DS/CDMA system," vol. 12, no. 5, pp. 796807, June 1994.

[12] S. Toumpis and A. J. Goldsmith, "Capacity regions for wireless ad hoc networks," IEEE Trans. Wireless Commun., vol. 2, no. 4, pp. 736 - 748, July 2003. 